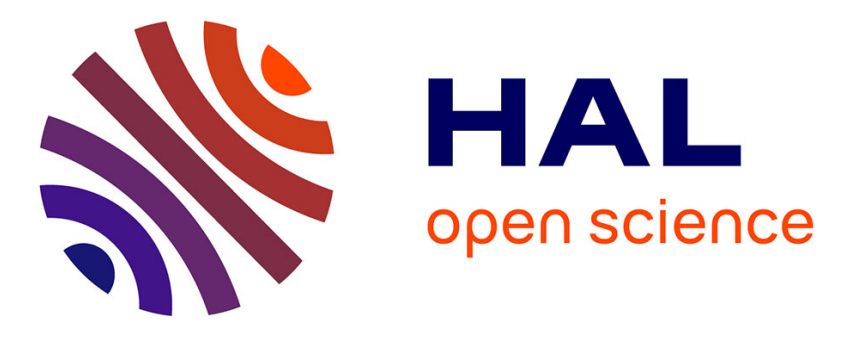

\title{
Combining Ellipsometry and AFM to Probe Sub-nanometric Precursor Films Dynamics of Polystyrene Melts
}

Claire Schune, Marc Yonger, Bruno Bresson, Laurent Guy, Christian Frétigny, Thomas Chaussée, François Lequeux, Hélène Montes, Emilie Verneuil

\section{To cite this version:}

Claire Schune, Marc Yonger, Bruno Bresson, Laurent Guy, Christian Frétigny, et al.. Combining Ellipsometry and AFM to Probe Sub-nanometric Precursor Films Dynamics of Polystyrene Melts. Langmuir, 2019, 10.1021/acs.langmuir.9b00768 . hal-02150026

\section{HAL Id: hal-02150026 \\ https://hal.science/hal-02150026}

Submitted on 6 Jun 2019

HAL is a multi-disciplinary open access archive for the deposit and dissemination of scientific research documents, whether they are published or not. The documents may come from teaching and research institutions in France or abroad, or from public or private research centers.
L'archive ouverte pluridisciplinaire HAL, est destinée au dépôt et à la diffusion de documents scientifiques de niveau recherche, publiés ou non, émanant des établissements d'enseignement et de recherche français ou étrangers, des laboratoires publics ou privés. 


\title{
Combining Ellipsometry and AFM to Probe Sub-nanometric Precursor Films Dynamics of Polystyrene Melts
}

\author{
Claire Schune, ${ }^{*} \dagger$ Marc Yonger ${ }^{\dagger}$ Bruno Bresson,${ }^{\dagger}$ Christian Fretigny, ${ }^{\dagger}$ Laurent \\ Guy,,$^{\ddagger}$ Thomas Chaussée, ${ }^{\ddagger}$ Franç̧ois Lequeux, ${ }^{\dagger}$ Hélène Montes, ${ }^{\dagger}$ and Emilie \\ Verneuil*, $^{*}$ \\ $\dagger$ Soft Matter Sciences and Engineering (SIMM), ESPCI Paris, PSL University, Sorbonne \\ Université, CNRS, F-75005 Paris, France \\ $\ddagger$ Solvay Silica, 15 rue Pierre Pä̈s, BP 52, F-69660 Collonges-au-Mont-d’Or, France \\ E-mail: claire.schune@espci.fr; emilie.verneuil@espci.fr
}

\begin{abstract}
We investigate the evolution over time of the space profiles of precursor films spreading away from a droplet of polymer, in the poorly explored pseudo-partial wetting case. We use polystyrene melt droplets on oxidized silicon wafers. Interestingly, the film thicknesses measured by ellispometric microscopy are found in a 0.01 to $1 \mathrm{~nm}$ range. These thicknesses were validated by atomic force microscopy (AFM) measurements performed on the textured film obtained after a quench in temperature. From this, an effective thickness is obtained and compares well to the thicknesses measured by ellipsometry, validating the use of an optical method in this range of thickness. Ellipsometric microscopy provides a height resolution below the ångström with lateral resolution, image size and framerate well adapted to spreading precursor films. From
\end{abstract}


this, we demonstrate that precursor films of polystyrene consist of polymer chains with a surface density decreasing to 0 away from the droplet. We further find that the polymer chains follow a simple diffusive law with diffusion coefficient independent of density. This demonstrate that polystyrene chains spread independently in precursor films in pseudo partial wetting condition. This behavior differs significantly from the case of chains spreading in total wetting, for which the diffusion coefficient was found in the literature to depend on surface density or thickness.

\section{Introduction}

When a liquid wets a surface, various situations may arise, depending on the surface energy of the liquid and the substrate. The most common situation is partial wetting, where the liquid droplet exhibits a non-zero contact angle on the bare solid surface at equilibrium. When the substrate is of higher surface energy than the liquid, total wetting or pseudo-partial wetting is expected, ${ }^{1,2}$ where a film of nanometer thickness spreads around the droplet. ${ }^{3}$ Total wetting is characterized by a zero contact angle at equilibrium, while the out of equilibrium situation corresponds to a precursor film spreading ahead of a spreading droplet. ${ }^{4,5}$ In pseudo-partial wetting, the equilibrium corresponds to a motionless droplet with a nonzero contact angle, which coexists with its precursor film. ${ }^{6-9}$ Theoretically, this latter case is expected when long range interactions between the liquid, substrate and vapor result in an effective attraction between the film interfaces. ${ }^{6}$ Studying the dynamics of the precursor films has been thought of as a way to characterize the substrate/molecules interactions and their

dynamics at interfaces, ${ }^{2}$ particularly for polymers for which confinement effects are expected to be enhanced. A careful look at the literature on the precursor films dynamics and morphology around droplets of polymer melts shows that existing works were actually limited to polymer/substrate pairs that were almost always in total wetting conditions, except for a small number of studies. ${ }^{7,8}$ When pseudo-partial wetting was identified experimentally, the precursor film profiles were however not resolved. Hence, the specific behavior of precursor 
films of polymer melts spreading around droplets in pseudo-partial wetting conditions is still an open question. Its elucidation would release some information on the effects of attractive long range interactions on confined polymer chains.

The case of polymer thin films experiencing attractive long range interactions were studied in depth in another experimental configuration: ${ }^{10-13}$ by observing the spontaneous dewetting patterns of homogeneous polystyrene films on oxidized silicon wafers prepared by spincoating. ${ }^{10,12}$ These previous studies demonstrated that this system experiences effective van der Waals interactions, that are attractive. They result in pseudo partial wetting condition, with an interaction potential that Seeman et al. determined. ${ }^{12}$ They also demonstrated that the final stage of dewetting consists in microdroplets separated by a residual polystyrene film or "equilibrium wetting layer", ${ }^{12,14}$ which can be destabilized into even smaller nanodroplets when quenched to lower temperatures. ${ }^{11}$ The dynamics of this interdroplets layer is not accessible in these configurations, and its thickness profile between microdroplets was not resolved. Instead, an average thickness was measured by x-ray reflectivity (XRR) at $1.3 \pm 0.3 \mathrm{~nm}$ for a molar mass $2000 \mathrm{~g} / \mathrm{mol}^{12}$ or $3.8 \pm 0.3 \mathrm{~nm}$ for $M_{w}=30600 \mathrm{~g} / \mathrm{mol}^{11,13}$ These lengthscales compare to the radius of gyration of the polymer $(1.2 \mathrm{~nm}$ and $4.5 \mathrm{~nm}$ respectively), raising the question of polymer confinement. ${ }^{13,15}$ For the smaller polymer, this thickness compares also to the typical monomer size, which interstingly raises the question of the density in the film, as pointed out by several other studies. ${ }^{2,16-18}$ Experimentally, it also questions the use of optical techniques on precursor films: what should be the optical index to be used to convert raw optical data into a length for sub-nanometer-thick, or, equivalently, dilute films? ${ }^{19}$

Imaging techniques such as atomic force microscopy (AFM), ${ }^{12,20-25}$ ellipsometry, ${ }^{5,17,26,27} \mathrm{X}$ ray reflectivity ${ }^{13,16,19}$ or surface plasmon resonance ${ }^{18}$ have greatly contributed to the understanding of precursor films behavior and wetting phenomena. For thicknesses in the nanometer range for which the determination of absolute thicknesses is challenging, combinations of several techniques were tentatively implemented. Mean thicknesses in between one 
and thirty nanometers were measured by AFM or XRR and compared to ellipsometry. ${ }^{19-21,28}$ A discrepancy was obtained and was attributed to the irreproducibility of the AFM tip surface from one experiment to another, to the liquid spreading on the AFM tip, ${ }^{20,21,28}$ or to a contamination between the measurements on the same sample. ${ }^{19}$ On the other hand, in the literature, combining ellipsometry or XRR and AFM proved to be successfull if ellipsometry or XRR were used to measure a mean thickness value, while AFM characterized the roughness with good accuracy, its thickness and lateral resolutions being in the nanometer range. Examples can be found on various systems ranging from microgels ${ }^{29}$ to glassy polymer thin films, ${ }^{30-32}$ nanodewetted polymer film ${ }^{13}$ or silicon oxide layers. ${ }^{33}$

But, at this point, to the best of our knowledge, the measurement by ellispometry of subnanometric absolute thicknesses remains an open question. This technique is however well adapted to the determination of thickness profiles which evolve over time, are non homogeneous, and extend over hundreds of micrometers. Ellipsometric microscopy is indeed non invasive, has a lateral resolution of typically $10 \mu \mathrm{m}$ - while XRR provides a $100 \mu \mathrm{m}$ lateral resolution at best, and the framerate can be as low as a few minutes. Moreover, ellipsometric microscopy offers absolute thickness measurements over lateral distances in the $100 \mu \mathrm{m}$ range, which can be difficult to obtain by AFM.

In order to resolve the detailed morphology of wetting precursor films in pseudo-partial wetting conditions at the sub-nanometer scale, it is offered to work with droplets of polystyrene melts deposited on high energy oxidized silicon wafers, which are known to exhibit pseudopartial wetting behavior, and to image the precursor film during its spreading ahead of the droplet. We will compare ellipsometry measurements with a non-optical technique of high resolution, the AFM. Care must be taken to ensure no polymer transfer to the AFM tip and no significant evolution of the sample occurs between measurements with the two techniques. Polystyrene (PS) of molar mass $\mathrm{Mn} \simeq 1000 \mathrm{~g} / \mathrm{mol}$ is chosen in this study: its glass transition temperature in bulk being of $T_{g}=10{ }^{\circ} \mathrm{C}$, this polymer spreads on silicon wafers at moderately high temperature $-T_{g}+70{ }^{\circ} \mathrm{C}-$ and can be imaged by AFM. In addition, 
quenching the system is likely to induce a texturing of the precursor film, as observed in the past on homogeneous thin polystyrene films, favouring its imaging by AFM. ${ }^{11}$

The purpose is to demonstrate the ability to measure the spreading dynamics of subnanometric wetting films by high resolution ellipsometry mapping. The dynamics of this model system will then be analysed and compared with a diffusion model, accounting for the density of polymers in the film.

\section{Materials and Methods}

\section{Sample}

The sample consists of a polystyrene droplet deposited on a silicon wafer, with an initial oxide layer of thickness $e$ ranging from 2 to 3.5 nanometers and measured by ellipsometry. The polystyrene is purchased from Sigma-Aldrich and characterized by chromatography: it has a molar mass $\mathrm{Mn}=920 \mathrm{~g} / \mathrm{mol}$ and a polydispersity index of 1.13. Its bulk glass transition temperature $T_{g}=10^{\circ} \mathrm{C}$ is measured by differential scanning calorimetry. The bulk refractive index of PS is $n=1.59 .{ }^{34}$

Silicon wafers are purchased from Siltronix Silicon Technologies. The latter are carefully cleaned in a fresh "piranha" solution $\left(\mathrm{H}_{2} \mathrm{SO}_{4}: \mathrm{H}_{2} \mathrm{O}_{2}(33 \%), 2: 1 \mathrm{v}: \mathrm{v}\right)$, rinsed several times with ultra pure water, dried under a flow of $\mathrm{N}_{2}$, and stored in a clean chamber. Before each experiment the silicon wafer surface is cleaned again with a thirty-minute UV/Ozone treatment. The water contact angle on these surfaces is zero. The clean wafer is placed in an hermetic cell to avoid contamination, with a clean nitrogen flux at a relative humidity of $11 \%$ at room temperature, set by a saturated lithium chloride aqueous solution. Two different thermostatic baths are used to set the substrate temperature. 


\section{Droplet of PS}

A thin needle is first dipped into heated PS. The glass transition of PS being around $10{ }^{\circ} \mathrm{C}$, the PS drop is viscous enough at room temperature to remain at the tip of the needle during its manipulation. The needle is then controlled with micrometer screws to enter the hermetic cell from the top, while sealing is ensured. When it approaches the wafer surface at $T_{+}=\mathrm{Tg}+70{ }^{\circ} \mathrm{C}$ the polymer becomes less viscous, drips, and a droplet transfers at the wafer surface. All ellipsometry measurements are performed in the hermetic cell. After a time $t_{q}$ at $T_{+}$the sample is quenched at $T_{-}=T_{g}+10{ }^{\circ} \mathrm{C}$. The switch from $T_{+}$to $T_{-}$is achieved within a few minutes. The quench time $t_{q}$ is either 35 min or 1 day in order to vary the lateral extension of the precursor film that spreads at $T_{+}$.

\section{Microscopic Ellipsometry}

The ellipsometer is the model Nanofilm EP3 purchased from Accurion. The light source is a Xenon lamp with a filter at $\lambda=590 \mathrm{~nm}$ and a bandwith of $10 \mathrm{~nm}$. The angle of incidence is fixed at $65^{\circ}$. The ellipsometer is equiped with a $5 \times$ objective and a camera yielding images of milimeter size, with a minimum time period of four minutes. The lateral resolution of the ellipsometer is around eight micrometers.

Ellipsometry is based on the measurement of the polarization state of reflected light. ${ }^{35,36}$ Formaly, it measures the ratio of the global reflexion coefficients of polarizations $\mathrm{p}$ and S written as $\rho=R_{p} / R_{s}=\tan \psi e^{i \Delta}$. For a stratified sample, the ellipsometry angles $\Delta$ and $\psi$ depend on the thickness and optical properties of each layer. A three-layer model (air/silica/silicon) is applied far from the droplet to measure the silicon oxide thickness at all times. In practice, it limits the experimental time to two days. A four-layer model (air/polymer/silica/silicon) is applied around the droplet to characterize the film. The optical indices we used are presented in Table 1. 
Table 1: Refractive indexes of the four materials taken into account in ellipsometric model.

\begin{tabular}{ccccc}
\hline & air & PS & silica & silicon \\
\hline optical index & 1 & 1.59 & 1.46 & $3.94 \pm 0.02 \mathrm{i}$ \\
\hline
\end{tabular}

In the range of film thicknesses $h$ between zero and one nanometer, we find a linear relation between $\Delta$ and $\psi$, while $\psi$ barely varies. The experimental $\Delta$ maps are compared with the results from the four-layer model to obtain thickness maps. In addition, noise reduction is achieved by taking advantage of the axial symmetry of the problem: h-averages on ten-degree arcs of one pixel width and centered at the droplet center are computed. Owing to the linear relation between $\Delta$ and $\psi$, averaging can be equivalently conducted on $\Delta$ or $h$.

\section{Ellipsometry Measurements of the Precursor Film at $T_{+}$}

A typical time series of mappings of ellipsometry angle $\Delta$ corresponding to the spontaneous spreading of PS is presented in Figure 1. A precursor film is observed to spread with axial symmetry around the droplet. The main droplet, shown as a half-circle, stops spreading after five minutes typically. At rest, its base radius $r_{d}$ can be precisely measured and ranges around $125 \mu \mathrm{m}$. Its volume is typically $10^{-10} \mathrm{~L}$. At $t_{q}=35 \mathrm{~min}$, the radial extension of the precursor film is around $70 \mu \mathrm{m}$ in this example (Figure 1d).

The motionless main droplet exhibits a constant non-zero contact angle while a film is seen to spontaneously spread around it, which clearly indicates that PS exhibits pseudopartial wetting condition on oxidized silicon. Macroscopic contact angle measurements on millimeter-sized droplets were also performed on a set-up described elsewhere. ${ }^{37}$ The main droplet exhibits an equilibrium contact angle $\theta_{\text {macro }}=8 \pm 3^{\circ}$ at $T_{+}$. Profiles in $\Delta$ as a function of the distance to the droplet edge $r-r_{d}$ can be extracted from the $\Delta$ maps at different times, as shown in Figure 1(e), where data are averaged over a ten-degree angular sector. 
(a)

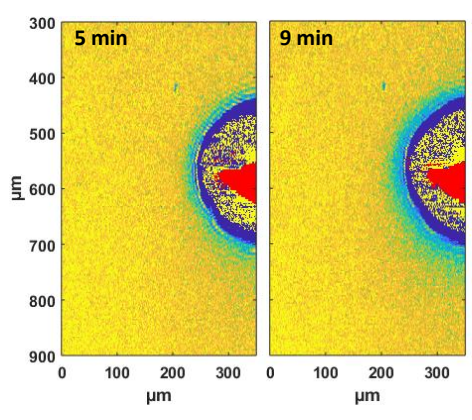

(c)

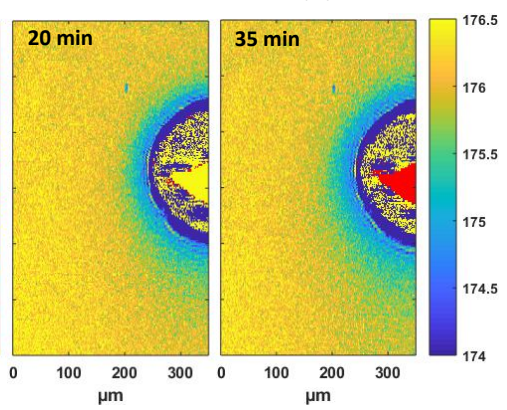

(d)

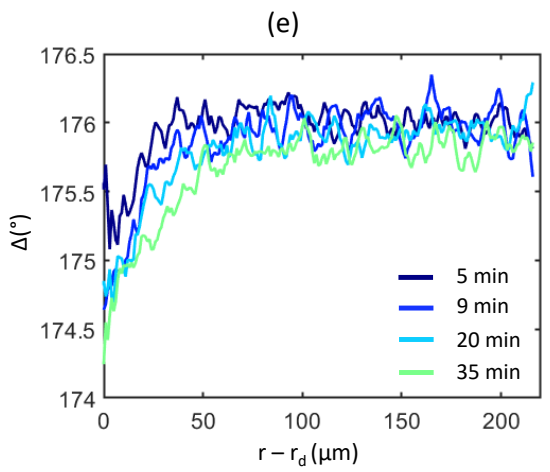

Figure 1: $\Delta$ maps measured by ellipsometric microscopy of a precursor film of PS at $T_{+}$ before quench $\left(t<t_{q}\right.$ ). (a) $t=5 \mathrm{~min}$. (b) $t=9 \mathrm{~min}$. (c) $t=20 \mathrm{~min}$. (d) $t=35 \mathrm{~min}$. Half-circle to the right: PS droplet with non zero contact angle. (e) $\Delta$-profiles as a function of the distance to the droplet edge $r-r_{d}$. The corresponding thickness mapping is presented Figure 7. Silica layer thickness $e=2.1 \mathrm{~nm}$ and quench time $t_{q}=35 \mathrm{~min}$.

Generally, in order to extract thickness maps from the ellipsometry angles $\Delta$, the latter values are compared to a theoretical model in which the polymer layer is considered as dense with an optical index equal to the bulk index. In the example of Figure 1, such an analysis yields thicknesses decreasing from around $1 \mathrm{~nm}$ to zero, as expected from the literature for interdroplet films. ${ }^{11,12}$ The polymer layer may therefore not be dense at large distance from the droplet edge: the surface may only be partially covered with polymer. It raises the question of using an effective medium framework to estimate the optical index, as suggested in the past. ${ }^{2}$

\section{AFM}

AFM images are obtained with a Bruker Icon microscope driven by a Nanoscope V controller.

The tip is a Budget Sensor tapping tip of typical frequency $300 \mathrm{kHz}$ and stiffness around $40 \mathrm{~N} / \mathrm{m}$. The tapping mode is used, with a frequency sweep of $1 \mathrm{~Hz}$. It has been checked that no polymer pollutes the tip, making the measurements reproducible. The background is flatten in the displayed images.

At $T_{+}$AFM shows that the film is not destabilized (no texture) but cannot provide an 
absolute measurement of its thickness. At $T_{-}$, the film destabilizes by spinodal dewetting. ${ }^{11}$ The onset of a texturing of the film allows to measure the polymer volume per unit area, or equivalently an effective thickness. The bulk glass transition temperature of our polystyrene being of ten degrees, the precursor film barely evolves at room temperature over the time needed to perform both the AFM and ellipsometry measurements, so that they can be compared. We take advantage of the axial symmetry of the precursor film and compare AFM and ellipsometry measurements at the same radial distance from the droplet edge, which can be precisely determined with both techniques, and at several angular values.

A three-step experimental method is next presented to validate precursor films thicknesses measured by ellipsometry at $T_{+}$. (i) Ellipsometry measurements are performed over time on the non destabilized films at $T_{+}=T_{g}+70^{\circ} \mathrm{C}$ - see Figure 1. (ii) The samples are quenched at $T_{-}=T_{g}+10^{\circ} \mathrm{C}$. Within a few hours the precursor films are observed to destabilized into an assembly of microdroplets. An effective thickness of the destabilized films is calculated from AFM measurements at $T_{-}$and compared to ellipometry measurements. (iii) The ellipsometry $\Delta$ profiles of the precursor film at $T_{+}$right before the quench, at $T_{-}$right after the quench, and a few hours later are compared.

Finally, the space and time variations of the polystyrene precursor films in pseudo-partial wetting will be measured and discussed in light of the literature on precursor films in total wetting conditions.

\section{Experimental Results}

\section{AFM and Ellipsometry Measurements of the Destabilized Precursor Film at $T_{-}$}

AFM measurements are performed at $T_{+}=T_{g}+70{ }^{\circ} \mathrm{C}$ and $T_{-}=T_{g}+10^{\circ} \mathrm{C}$ on the precursor film to image its destabilization and catch the emergence of the microdroplets. Typical observations are presented in Figure 2. It is seen that the film starts destabilizing a few hours 
after quenching from $T_{+}$to $T_{-}-$Figure 2(c). The z colorbar has been purposely saturated at a maximal height of twenty nanometers to clearly see the microdroplets appearing. No structures can be resolved at $T_{+}$before the quench - Figure 2(a), nor after reheating the quenched destabilized film from $T_{-}$to $T_{+}$(not shown). This demonstrates that the texturing is reversible.

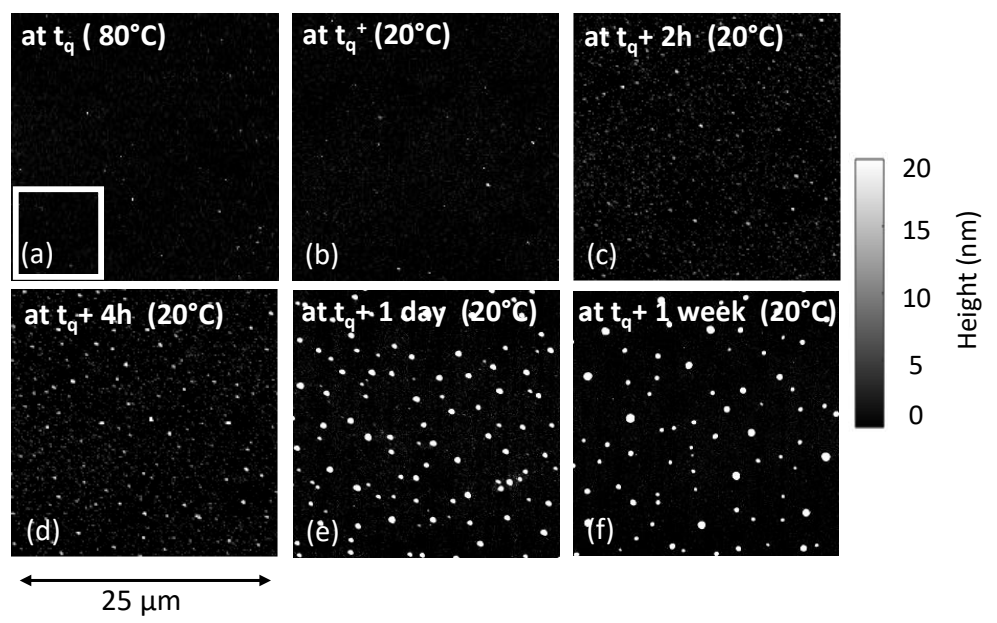

Figure 2: AFM height measurements of a precursor film of PS. The radial distance between the main droplet edge and the center of the image is $r-r_{d}=40 \mu \mathrm{m}$. The white square represents the eight-micrometer lateral resolution of the ellipsometer. The main droplet is located to the left of the images. (a) at $T_{+}=T_{g}+70{ }^{\circ} \mathrm{C}$ and at time $t_{q}$ : no structures are resolved. (b-f) at $T_{-}=T_{g}+10^{\circ} \mathrm{C}$ and for different times after the quench. The silica layer thickness is $e=3.3 \mathrm{~nm}$ and $t_{q}=1$ day.

Ellipsometry measurements are also performed at different times after the quench at $T_{-}$ as shown in Figure 3. These thickness maps are obtained assuming the PS layer can be described using bulk PS optical index, a choice that we will justify. The emergence of a texturing of the film is clearly visible at late times - Figure 3(b). 
(a)

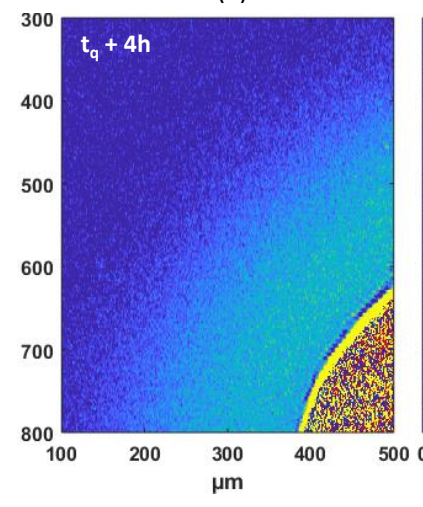

(b)

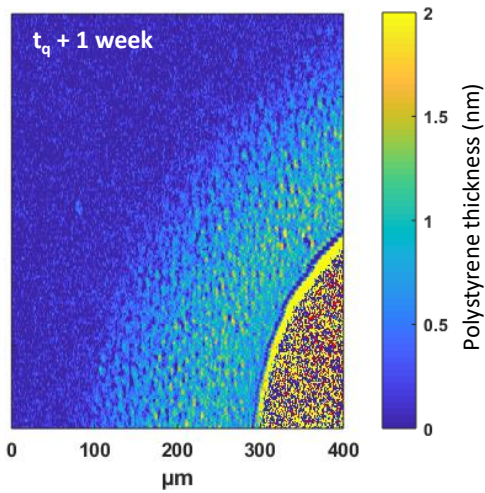

Figure 3: Thickness maps measured by ellipsometric microscopy of a precursor film of PS after the quench at $T_{-}=T_{g}+10{ }^{\circ} \mathrm{C}$. (a) $t=t_{q}+4 \mathrm{~h}$. (b) $t=t_{q}+1$ week. A part of the main droplet is visible at the bottom right corner. Silica layer thickness $e=2.6 \mathrm{~nm}$; Quench time $t_{q}=1$ day.

The AFM measurements at $T_{-}=T_{g}+10{ }^{\circ} \mathrm{C}$ on the same destabilized film associated to Figure 3 are presented in Figure 4, at various distances $r-r_{d}$ from the main droplet edge. After a few hours after quench and at all distances from the main droplet, the destabilized film consists in an assembly of microdroplets. Higher resolution images of microdroplets were also performed by AFM (not shown) from which a typical microdroplet profile is extracted (Figure 4d). All microdroplets can be fitted to a parabola with axial symmetry thus demonstrating they are spherical caps. They form a contact angle $\theta_{\text {micro }}=17 \pm 3^{\circ}$ at late stages of destabilization. For each microdroplet the base radius $r_{\text {micro }}$ and the maximal height $h_{\max }$ are measured, as defined in Figure 4(d). Average values are calculated over the area of each image $-25 \times 12.5 \mu \mathrm{m}^{2}, 25 \times 25 \mu \mathrm{m}^{2}$ or $10 \times 10 \mu \mathrm{m}^{2}-$ and the obtained values $\bar{r}_{\text {micro }}$ and $\bar{h}_{\text {max }}$ are detailed in Table 2. $N$ is the number of microdroplets per square micrometer and $\mathcal{R} \sim \bar{r}_{\text {micro }}^{2} / 2 \bar{h}_{\text {max }}$ is an indicative radius of curvature calculated from the average radius and height considering small aspect ratios $h_{\max } / r_{\text {micro }}$. 
(a)

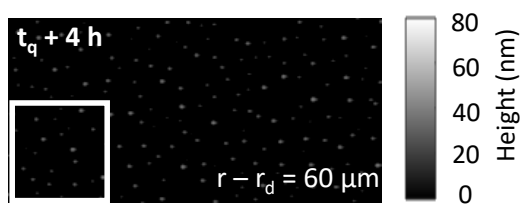

(b)

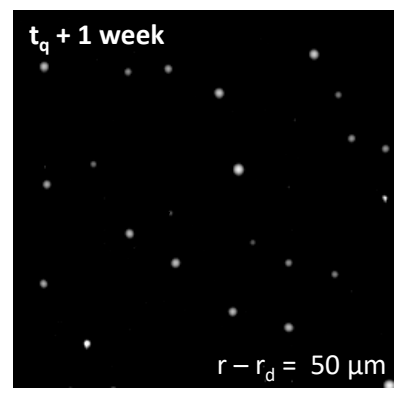

(c)

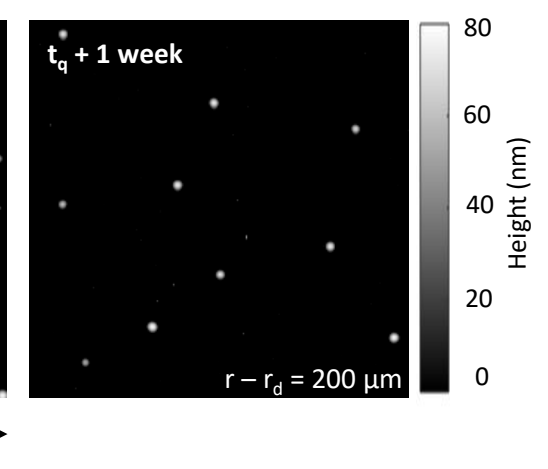

(d)

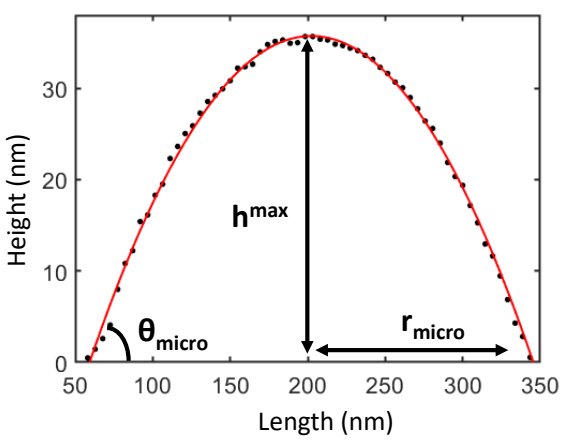

$25 \mu \mathrm{m}$

Figure 4: AFM height measurements of a precursor film of PS at $T_{-}=T_{g}+10{ }^{\circ} \mathrm{C}$. The images are $25 \mu \mathrm{m}$ wide and the white square represents the eight-micrometer lateral resolution of the ellipsometer. The main droplet is located to the left of the images. (a) at $t=t_{q}+4 \mathrm{~h}$ and $r-r_{d}=60 \mu \mathrm{m}$. (b) at $t=t_{q}+1$ week and $r-r_{d}=50 \mu \mathrm{m}$. (c) at $t=t_{q}+1$ week and $r-r_{d}=200 \mu \mathrm{m}$. (d) Height profile of a microdroplet fitted to a parabola: all microdroplets are spherical caps with good accuracy, with a contact angle $\theta_{\text {micro }}$, a base radius $r_{\text {micro }}$ and a maximal height $h_{\max } . t_{q}=1$ day.

Table 2: Characteristics of the microdroplets of PS after quenching the sample at $T_{-}$. These data correspond to the images of Figure $4 . \bar{r}_{\text {micro }}$ is the average of the radii of the microdroplets at a given distance from the main droplet edge, $\bar{h}_{\max }$ the average maximum height, $N$ the number of microdroplets per square micrometer and $\mathcal{R}$ an indicative radius of curvature.

\begin{tabular}{cccc}
\hline & $t_{q}+4 \mathrm{~h}$ & $t_{q}+1$ week & $t_{q}+1$ week \\
& $r-r_{d}=60 \mu \mathrm{m}$ & $r-r_{d}=50 \mu \mathrm{m}$ & $r-r_{d}=200 \mu \mathrm{m}$ \\
\hline $\bar{r}_{\text {micro }}(\mathrm{nm})$ & 200 & 390 & 380 \\
$\bar{h}_{\text {max }}(\mathrm{nm})$ & 25 & 55 & 69 \\
$N\left(\mu m^{-2}\right)$ & 0.67 & 0.04 & 0.02 \\
$\mathcal{R}(\mathrm{nm})$ & 800 & 1380 & 1050 \\
\hline
\end{tabular}

From Figure 2, Figure 4 and Table 2, two observations can be made. First, the microdroplets morphology evolves over time at a given distance $r-r_{d}$ from the main droplet edge. While the contact angle $\theta_{\text {micro }}$ seems to be preserved, the base radius and height increase over time. A decrease by more than a factor of ten of the density of microdroplets $N$ is ob- 
served between Figure 4(a) and (b). Secondly, at a given time, the number of microdroplets $N$ decreases with the distance to the main droplet.

As seen in Figure $4(\mathrm{a})$, at $t_{q}+4 \mathrm{~h}$ with $t_{q}=1$ day the typical distance between the microdroplets is way smaller than the ellipsometer lateral resolution, depicted by the white square. On the contrary, at $t_{q}+1$ week the interdroplets distance compares with the ellipsometer resolution. The appearance of a texturing on the destabilized film on the ellipsometry maps of Figure 3 can thus be related to the spatial distribution of the microdroplets.

The effective thickness $h_{A F M}$ of the destabilized precursor film at a given distance from the droplet edge can be calculated from the AFM measurements by integrating the height maps on each image to obtain the total volume and dividing it by the surface of the image.

\section{Discussion}

\section{Comparison of AFM and Ellipsometry Measurements on the Desta-}

\section{bilized Film at $T_{-}$}

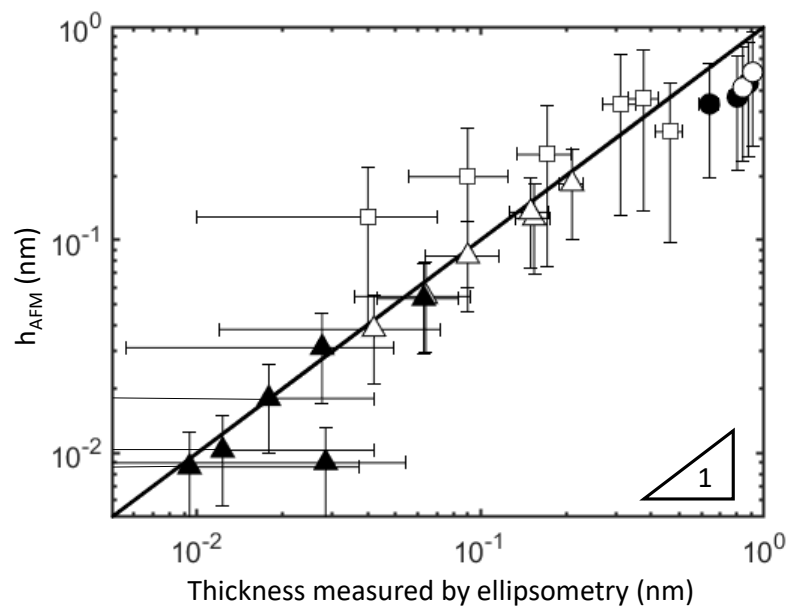

Figure 5: Thickness of the destabilized PS film obtained from the AFM measurements as a function of the thickness measured by ellipsometry at $T_{-}=T_{g}+10{ }^{\circ} \mathrm{C}$ considering a dense PS layer. $\circ t_{q}=1$ day and $t=t_{q}+4 \mathrm{~h} ; \bullet t_{q}=1$ day and $t=t_{q}+1$ week ; $\triangle t_{q}=35$ min and $t=t_{q}+1$ day ; $\boldsymbol{\Delta} t_{q}=35$ min and $t=t_{q}+1$ week ; $\square t_{q}=1$ day and $t=t_{q}+6 \mathrm{~h}$. Black line: $y=x$. 
The ellipsometry measurements performed after the quench on the destabilized film and analyzed assuming a dense layer of PS are compared to the AFM effective thickness $h_{A F M}$ in Figure 5. The data scattering can be understood considering two effects. First, the effective thickness $h_{A F M}$ is affected by statistical effects in the AFM imaging of microdroplets. It indeed depends on a compromise between image size and pixel resolution, within the limit of short experimental time to avoid ripenning. Secondly, ellipsometric microscopy cannot resolve the microdroplets and the fluctuations of the ellipsometry measurements are related to the statistics on the microdroplets in the precise area where the ellipsometry averages are performed. These two effects are accounted for in the error bars of Figure 5.

Finally, AFM effective thickness and ellipsometry thickness on the destabilized precursor film are found to be in fair agreement in Figure 5. This result suggests that even though the lateral resolution of the ellipsometer does not allow to resolve the microdroplets, the thickness measured by ellipsometry accounts for the microdroplets volume fraction and hence can be interpreted as an effective thickness with optical index equal to the bulk. For small volume fraction, this effective layer may be as thin as $0.1 \AA$.

This can be understood within the framework of an effective medium hypothesis. It is indeed possible to evaluate an effective optical index $n_{e f f}$ for the destabilized PS film via the Maxwell-Garnett relation. ${ }^{38}$ In a simple view, the microdroplets of base radius $r_{\text {micro }}$ can be reshaped into spheres of radius $R_{\text {micro }}$ with the same volume. The characteristic size of these spheres $R_{\text {micro }}$ being smaller than the wavelength used for the ellipsometric measurements, the relation between the effective relative permittivity $\epsilon_{e f f}$ of the destabilized film and the relative permittivity of PS $\epsilon_{P S}$ is given by Equation 1, where the volume fraction of the microdroplets is $f$ and the relative permittivity of air is $\epsilon_{\text {air }}$.

$$
\frac{\epsilon_{e f f}-\epsilon_{a i r}}{\epsilon_{e f f}+2 \epsilon_{a i r}}=f \frac{\epsilon_{P S}-\epsilon_{a i r}}{\epsilon_{P S}+2 \epsilon_{a i r}}
$$

In our case, we find $f \sim 10^{-2}<<1$. The characteristic distances between the spheres being 
always significantly smaller than their radius $R_{\text {micro }}$, the structure factor does not play a significant role and the approximation of spherical PS particules rather than spherical caps holds. Because the ratio $f .\left(\epsilon_{P S}-\epsilon_{\text {air }}\right) /\left(\epsilon_{P S}+2 \epsilon_{\text {air }}\right)<<1$, Equation 1 simplifies into:

$$
n_{e f f}=\sqrt{\epsilon_{e f f}} \simeq n_{a i r}\left(1+\frac{3}{2} f \frac{\epsilon_{P S}-\epsilon_{a i r}}{\epsilon_{P S}+2 \epsilon_{\text {air }}}\right)
$$

It can then theoretically be shown that in the model used to convert the $\Delta$ maps into thicknesses, it is equivalent to consider (i) a layer of PS microdroplets of thickness $h_{\max }$ and low volume fraction $f$ with an effective optical index $n_{\text {eff }}$ given by Equation 2 or (ii) a layer of effective thickness $h_{\text {eff }}$ around one nanometer having the optical index of bulk PS. This effective medium analysis therefore rationalizes our experimental results, where effective thicknesses below $0.1 \mathrm{~nm}$ are measured (Figure 5).

Altogether, the thicknesses measured by ellipsometry on a destabilized film at $T_{-}$can be trusted and will be compared to thicknesses at $T_{+}$where the precursor film is not destabilized.

\section{Comparison of Ellipsometry Measurements on the Film at $T_{+}$and on the Destabilized Film at $T_{-}$}

The ellipsometry effective thickness profiles of the precursor film of PS right before the quench when it is not destabilized, and after the quench are compared in Figure 6, using the bulk optical index of PS. The profiles remarkably superimpose, highlighting the polymer volume conservation in the precursor film at $T_{+}$and its destabilized form at $T_{-}$. As mentioned earlier, a linear relation holds between $\Delta$ and $h$ in the range of thicknesses at stake here, indicating than no other values than the bulk for the optical index of PS would give a superimposition of the profiles. In light of this observation and the results of previous sections, the model implemented considering the bulk optical index of PS can also be used at $T_{+}=T_{g}+70{ }^{\circ} \mathrm{C}$ to convert the $\Delta$ maps into effective thicknesses, despite subnanometric thicknesses. 


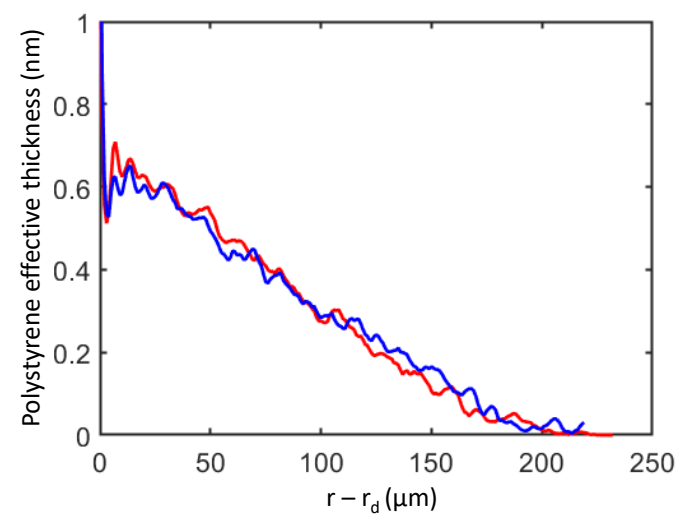

Figure 6: Effective thickness profiles of a precursor film of PS measured by ellipsometric microscopy: (red) right before the quench at $T_{+}=T_{g}+70{ }^{\circ} \mathrm{C}$; (blue) after the quench, at $t_{q}+4 \mathrm{~h}$ and $T_{-}=T_{g}+10{ }^{\circ} \mathrm{C}$ when the film is destabilized. The bulk optical index value of $\mathrm{PS}$ is implemented in the four-layer model. Silica layer thickness $e=2.2 \mathrm{~nm}$; Quench time: $t_{q}=1$ day.

\section{Structure of the Polystyrene Films at $T_{-}$}

Let us first discuss the morphology of the precursor film when quenched at ambient temperature. The destabilized film is seen to form microdroplets (Figure 4). The time evolution of the microdroplets (see Table 2) indicates that some polymer molecules may remain at the surface between the microdroplets allowing for the observed mass transfer between them. These transfers could be driven by Laplace pressure gradients causing the smaller microdroplets to empty into the bigger ones and in the main droplet. The typical Laplace pressure in a microdroplet is $P_{L} \sim 2 \gamma / \mathcal{R} \simeq 10^{4} \mathrm{~Pa}$, where $\gamma \simeq 30 \mathrm{mN} / \mathrm{m}$ is the surface tension of PS. ${ }^{12}$ As $\mathcal{R}$ decreases with distance, $P_{L}$ increases accordingly, resulting in a pressure gradient. Such a ripenning mechanism could account for the observed increase of the mean radius of curvature over time. Besides, no polymers is resolved by AFM between the microdroplets. These observations tend to show that the quenched film consists of bulk liquid microdroplets coexisting with a very dilute film in which polymers are mobile.

The formation mechanism of the microdroplets of polystyrene is not the main topic of this 
paper, but a few words can be said. The spinodal dewetting of thin polystyrene films from oxidized silicon substrates when increasing the temperature has been extensively measured in the literature ${ }^{12,39-41}$ in the case of spincoated films. At high temperature, a film coexisting with an assembly of droplets of various sizes and their spatial distribution was reported. These spincoated films are initially out of equilibrium, and evolve as soon as mobility is given by increasing the temperature. In the present study, the precursor film spreads spontaneously on the silicon wafer at high temperature and does not compare to the literature cited above. However, a relevant comparison with the studies by Muller-Buschbaum et al. can be made. They showed that very thin interdroplet films obtained by spinodal dewetting destabilize themselves through a secondary spinodal dewetting mechanism when quenched down to lower temperature. ${ }^{11}$ Our observations are therefore consistent with the studies of Muller-Buschbaum et al., although no descriptions of the effects of the temperature on the equilibrium states of the polymer films are available. The temperature dependence of the energy landscape is further confirmed by observation of the reversibility of the film texturing with temperature.

\section{Structure of the Polystyrene Films at $T_{+}$}

We now discuss the morphology of the polystyrene films obtained at high temperature. As seen in Figure 6, the precursor film effective thickness is found to decrease continuously from $h_{1}=0.6 \mathrm{~nm}$ to zero. In bulk, the radius of gyration is $R_{g} \sim 0.9 \mathrm{~nm}$ and the Kühn length for polystyrene is $b=1.8 \mathrm{~nm}$. The effective thicknesses in the precursor film are therefore always smaller than both $R_{g}$ and $b$. In addition, no texturing is imaged by AFM at $T_{+}$, neither before the quench nor after reheating of the quenched destabilized film. These results indicate that the precursor film, which spontaneously spreads around droplets on a semi-infinite substrate, may not be dense when attractive long range interactions are at play. Instead, its density decreases away from the droplet, which acts as a reservoir. The thickness of the film 
$h_{\text {eff }}$ corresponds to the molecular volume $v$ times the chain surface density $n: h_{e f f}=n v$.

Finally, our results show that the presursor films spreading ahead a macroscopic droplet in pseudo-partial wetting condition exhibit a very peculiar profile that is reported here for the first time: the chain surface density decreases towards 0 away from the motionless droplet. In total wetting, or equivalently when long range interactions are repulsive, precursor films were shown to be very different: they exhibit a pancake-like mode of spreading with an almost flat precursor film of quasi-uniform density, ${ }^{16}$ ending with a sharp step at its outermost end. ${ }^{2,27,42-44}$

\section{Dynamics of the Spreading Polystyrene Film}

(a)

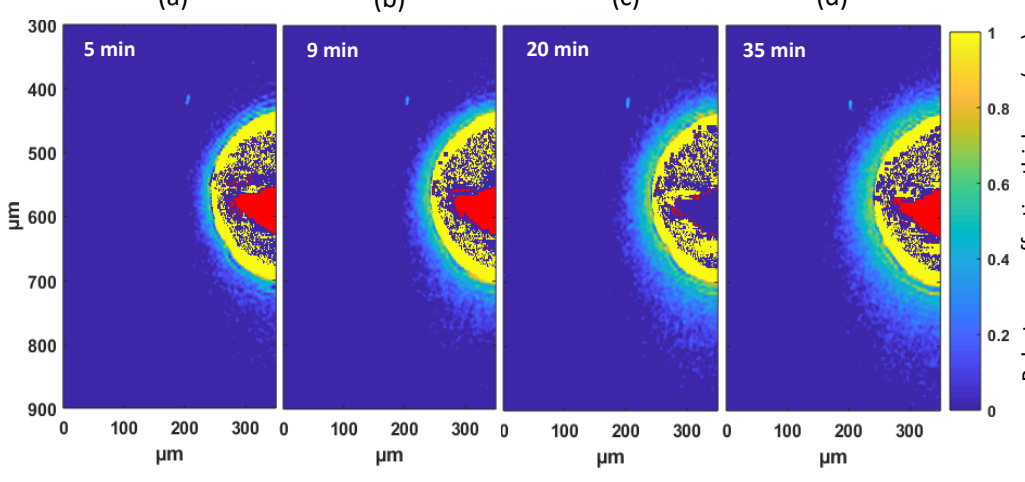

(e)

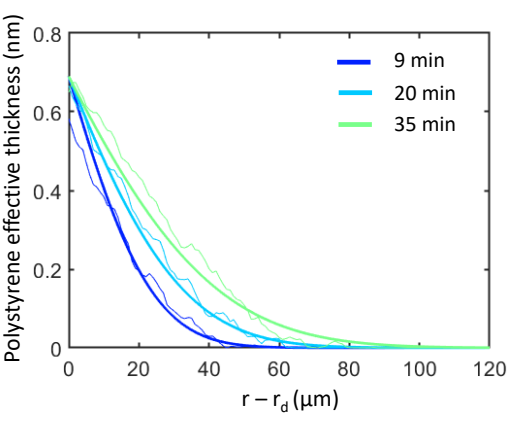

Figure 7: Effective thickness maps measured by ellipsometric microscopy of a precursor film of PS at $T_{+}$before quench $\left(t<t_{q}\right.$ ). (a) $t=5 \mathrm{~min}$. (b) $t=9 \mathrm{~min}$. (c) $t=20 \mathrm{~min}$. (d) $t=35$ min. The non-zero contact angle droplet is visible. (e) Corresponding height profiles, as a function of the distance to the droplet edge $r-r_{d}$. Lines: fit to solutions of Equation 3 with $D=0.32 \mu \mathrm{m}^{2} / \mathrm{s}$ and $h_{1}=0.69 \mathrm{~nm}$. The silica layer thickness is $e=2.1 \mathrm{~nm}$ and $t_{q}=35 \mathrm{~min}$.

The spreading dynamics of the precursor film at high temperature $T_{+}$will now be precisely measured. The $\Delta$ maps of Figure 1 are converted into effective thicknesses in Figure 7 . It is observed that at the droplet edge $r_{d}$, the film thickness $h_{1}$ is constant and ranges around one nanometer on all experiments. Therefore, hypothesis is made that the film is pinned to the main droplet edge at a height $h_{1}$. A diffusion model for the polymer molecules with 
a diffusion coefficient $D$ independent of the thickness can be derived and compared to the data. The diffusion equation is presented in Equation (3).

$$
\frac{\partial h_{e f f}}{\partial t}=\frac{1}{r} \frac{\partial}{\partial r}\left(r D \frac{\partial h_{e f f}}{\partial r}\right), h_{e f f}\left(r=r_{d}, t\right)=h_{1} \text { and } h_{e f f}\left(r>r_{d}, t=0\right)=0
$$

We emphasize that $h_{\text {eff }}$ represents the product of chain surface density to the molecular volume. Solution profiles $h_{\text {eff }}(r, t)$ to Equation (3) are fitted to the experimental data in Figure 7(e). The polymer chains in the precursor film are found to exhibit a diffusive dynamics with $D=0.32 \pm 0.05 \mu \mathrm{m}^{2} / \mathrm{s}$ and $h_{1}=0.69 \pm 0.02 \mathrm{~nm}$. This shows that the diffusion coefficient of the polymer chains is independent of their surface density. Hence, for the first time, we demonstrate that a simple diffusive behavior describes the spreading dynamics of polymer chains in precursor films exhibiting pseudo-partial wetting condition. The fact that $D$ is found to be independent of the chain surface density suggests that the polymer chains diffuse independently from one another in this case.

Remarkably, both the structure and the dynamics of the present polystyrene precursor films are different from the ones observed in total wetting condition. Indeed, in past studies where polymer melts and substrates exhibited total wetting conditions, the precursor films were measured to be flat, of quasi-uniform thickness in the nanometer range, ending with a sharp downward step. ${ }^{2}$ The lateral extent of these precursor films was defined as the distance $L$ between the droplet edge and this step. A diffusive-like behavior of the type $L \sim \sqrt{(} D t)$ was often observed. ${ }^{18,27,43-47}$ From this, an effective diffusion coefficient was introduced, consistently with theoretical predictions, ${ }^{48,49}$ which however characterizes the film tip only. The existence of such a pancake-shaped precursor film together with its time evolution implies a thickness or density-dependent diffusion coefficient. This result was established in studies on systems exhibiting total wetting conditions, ${ }^{18,50-53}$ and accounted for by theoretical models summarized in Section 5 of the topical review by Popescu et al. ${ }^{2}$ This suggests that chains 
in precursor films corresponding to total wetting, or equivalently experiencing repulsive long range interactions, do not diffuse independently from one another.

\section{Conclusions}

In this paper, the wetting of polystyrene melts on oxidized silicon wafers is investigated. Polystyrene exhibits pseudo-partial wetting condition on oxidized silicon wafers at high temperature: a motionless macroscopic droplet coexists with a spreading precursor film. Ellipsometric microscopy is used to achieve high resolution effective thickness measurements of the film at high and room temperatures. The effective thickness profiles obtained are compared with AFM height measurements to validate the thickness mappings obtained by ellipsometry at a sub-nanometer scale: effective thicknesses below the ångström can be measured. To this end, advantage is taken from the destabilization of the film when quenched at room temperature. When destabilized, the precursor film consists of an assembly of microdroplets, with few polymer chains remaining in between, causing inter-droplet transfers due to Laplace pressure gradients. At high temperature, however, these microdroplets do not form.

We show that in pseudo-partial wetting condition the polystyrene precursor film presents a chain surface density that continuously decreases towards zero away from the contact line. Its dynamics can be described by a remarkably simple model: a diffusive law with a diffusion coefficient $D$ independent of thickness or surface density. This result shows that in pseudo-partial wetting condition, polystyrene precursor films consist in chains crawling independently from one another, contrary to the total wetting case where precursor films are known to spread in a pancake-like mode. We anticipate that measuring the structure and dynamics of precursor films of polymer melts in pseudo-partial wetting configuration will allow to characterize the dynamics of isolated chains on surfaces. 


\section{References}

(1) Bonn, D.; Eggers, J.; Indekeu, J.; Meunier, J. Wetting and spreading. Reviews of Modern Physics 2009, 81, 739-805.

(2) Popescu, M. N.; Oshanin, G.; Dietrich, S.; Cazabat, A. M. Precursor films in wetting phenomena. Journal of Physics Condensed Matter 2012, 24, 1-51.

(3) Hardy, W. III. The spreading of fluids on glass. London, Edinburgh, Dublin Phil. mag. J. Sci. 1919,

(4) de Gennes, P. G. Statics and dynamics. Reviews of Modern Physics 1985, 57, 827.

(5) Ausserré, D.; Picard, A. M.; Léger, L. Existence and role of the precursor film in the spreading of polymer liquids. Physical Review Letters 1986, 57, 2671-2674.

(6) Brochard-Wyart, F.; di Meglio, J. M.; Quéré, D.; de Gennes, P. G. Spreading of Nonvolatile Liquids in a Continuum Picture. Langmuir 1991, 7, 335-338.

(7) Silberzan, P.; Léger, L. Evidence for a new spreading regime between partial and total wetting. Physical Review Letters 1991, 66, 185-188.

(8) Esibov, L.; Sarkisov, D.; Jeng, U.-S.; Crow, M. L.; Steyerl, A. Dynamics of pseudopartial wetting studied by neutron reflectometry. Physica B: Condensed matter 1998, 241-243, 1077-1079.

(9) Du, L.; Bodiguel, H.; Cottin, C.; Colin, A. Dynamics of liquid-liquid menisci in pseudopartial wetting. Chemical Engineering and Processing: Process Intensification 2013, $68,3-6$.

(10) Reiter, G. Unstable Thin Polymer Films: Rupture and Dewetting Processes. Langmuir 1993, 9, 1344-1351. 
(11) Muller-Buschbaum, P.; Vanhoorne, P.; Scheumann, V.; Stamm, M. Observation of nano-dewetting structures. Europhysics Letters 1997, 40, 655-660, WOS:000071087400012.

(12) Seemann, R.; Herminghaus, S.; Jacobs, K. Dewetting patterns and molecular forces: A reconciliation. Physical Review Letters 2001, 86, 5534-5537.

(13) Muller-Buschbaum, P.; Bauer, E.; Wunnicke, O.; Stamm, M. The control of thin film morphology by the interplay of dewetting, phase separation and microphase separation. Journal of Physics-Condensed Matter 2005, 17, S363-S386, WOS:000228031100008.

(14) Seemann, R.; Herminghaus, S.; Jacobs, K. Gaining control of pattern formation of dewetting liquid films. Journal of Physics-Condensed Matter 2001, 13, 4925-4938.

(15) Jiang, N.; Wang, J.; Di, X.; Cheung, J.; W., Z.; Endoh, M.; T., K.; Satija, S. K. Nanoscale adsorbed structures as a robust approach for tailoring polymer film stability. Soft Matter 2016, 12, 1801-1809.

(16) Daillant, J.; Benattar, J.; Bosto, L.; Leger, L. Final Stages of Spreading of Polymer Droplets on Smooth Solid Surfaces. Europhysics Letters 1988, 6, 431-436.

(17) Heslot, F.; Cazabat, A. M.; Fraysse, N. Diffusion-controlled wetting films. Journal of Physics: Condensed Matter 1989, 1, 5793-5798.

(18) Albrecht, U.; Achim, O.; Leiderer, P. Two-Dimensional Liquid Polymer Diffusion: Experiment and Simulation. PRL 1992, 68, 3192-3196.

(19) Toney, M. F.; Mate, C. M.; Leach, K. A.; Pocker, D. Thickness measurements of thin perfluoropolyether polymer films on silicon and amorphous-hydrogenated carbon with X-ray reflectivity, ESCA and optical ellipsometry. Journal of Colloid and Interface Science 2000, 225, 219-226. 
(20) Mate, C. M.; Lorenz, M. R.; Novotny, V. J. Atomic force microscopy of polymeric liquid films. The Journal of Chemical Physics 1989, 90, 7550-7555.

(21) Mate, C. M.; Novotny, V. J. Molecular conformation and disjoining pressure of polymeric liquid films. The Journal of Chemical Physics 1991, 94, 8420-8427.

(22) Glick, D.; Thiansathaporn, P.; Superfine, R. In situ imaging of polymer melt spreading with a high-temperature atomic force microscope. Applied Physics Letters 1997, 71, $3513-3515$.

(23) Villette, S.; Valignat, M. P.; Cazabat, A. M.; Schabert, F. A.; Kalachev, A. Ultrathin liquid films. Ellipsometric study and AFM preliminary investigations. Physica A: Statistical Mechanics and its Applications 1997, 236, 123-129.

(24) Xu, H.; Shirvanyants, D.; Beers, K.; Matyjaszewski, K.; Rubinstein, M.; Sheiko, S. S. Molecular motion in a spreading precursor film. Physical Review Letters 2004, 93, 1-4.

(25) Xu, L.; Salmeron, M.; Bardon, S. Wetting and molecular orientation of 8cb on silicon substrates. Physical Review Letters 2000, 84, 1519-1522.

(26) Leger, L.; Erman, M.; Guinet-Picard, A. M.; Ausserre, D.; Strazielle, C. Precursor film profiles of spreading liquid drops. Physical Review Letters 1988, 60, 2390-2393.

(27) Heslot, F.; Cazabat, A. M.; Levinson, P. Dynamics of wetting of tiny drops: Ellipsometric study of the late stages of spreading. Physical Review Letters 1989, 62, 1286-1289.

(28) Mate, C. M.; Lorenz, M. R.; Novotny, V. J. Determination of Lubricant Film Thickness on a Particulate Disk Surface by Atomic Force Microscopy. IEEE Transactions on Magnetics 1990, 26, 1225-1228.

(29) Schmidt, S.; Motschmann, H.; Hellweg, T.; von Klitzing, R. Thermoresponsive surfaces by spin-coating of PNIPAM-co-PAA microgels: A combined AFM and ellipsometry study. Polymer 2008, 49, 749-756. 
(30) Fian, A.; Haase, A.; Stadlober, B.; Jakopic, G.; Matsko, N. B.; Grogger, W.; Leising, G. AFM, ellipsometry, XPS and TEM on ultra-thin oxide/polymer nanocomposite layers in organic thin film transistors. Analytical and Bioanalytical Chemistry 2008, 390, $1455-1461$.

(31) Petri, D. F. Characterization of spin-coated polymer films. Journal of the Brazilian Chemical Society 2002, 13, 695-699.

(32) Sharma, S.; Johnson, R. W.; Desai, T. A. Evaluation of the Stability of Nonfouling Ultrathin Poly(ethylene glycol) Films for Silicon-Based Microdevices. Langmuir 2004, 20, 348-356.

(33) Fang, S. J.; Chen, W.; Yamanaka, T.; Helms, C. R. Influence of Interface Roughness on Silicon Oxide Thickness Measured by Ellipsometry. Society 1997, 144, 231-233.

(34) Sultanova, N.; Kasarova, S.; Nikolov, I. Dispersion properties of optical polymers. Acta Physica Polonica A 2009, 116, 585-587.

(35) Azzam, R.; Bashara, N. Ellipsometry and polarized light; Elsevier: Amsterdam, 1978; pp xvii +539 .

(36) Tompkins, H.; Irene, E. A. Handbook of ellipsometry; 2005; p 329.

(37) Dupas, J.; Verneuil, E.; Ramaioli, M.; Forny, L.; Talini, L.; Lequeux, F. Dynamic wetting on a thin film of soluble polymer: Effects of nonlinearities in the sorption isotherm. Langmuir 2013, 29, 12572-12578.

(38) Aspnes, D. Local-field effects and effective-medium theory: a microscopic perspective. American Journal of Physics 1982, 50, 704-708.

(39) Reiter, G. Dewetting of highly elastic thin polymer films. Physical Review Letters 2001, $8 \%, 186101-1-186101-4$. 
(40) Glynos, E.; Frieberg, B.; Green, P. F. Wetting of a multiarm star-shaped molecule. Physical Review Letters 2011, 10\%, 1-5.

(41) Seemann, R.; Herminghaus, S.; Neto, C.; Schlagowski, S.; Podzimek, D.; Konrad, R.; Mantz, H.; Jacobs, K. Dynamics and structure formation in thin polymer melt films. Journal of Physics Condensed Matter 2005, 17.

(42) Voué, M.; Valignat, M. P.; Oshanin, G.; Cazabat, A. M.; De Coninck, J. Dynamics of Spreading of Liquid Microdroplets on Substrates of Increasing Surface Energies. Langmuir 1998, 14, 5951-5958.

(43) Fraysse, N.; Valignat, M.; Cazabat, A.; Heslot, F.; Levinson, P. The Spreading of Layered Microdroplets. Journal of Colloid and Interface Science 1993, 158, 27-32.

(44) Mate, C. M. Anomalous diffusion kinetics of the precursor film that spreads from polymer droplets. Langmuir 2012, 28, 16821-16827.

(45) O’Connor, T. M.; Back, Y. R.; Jhon, M. S.; Min, B. G.; Yoon, D. Y.; Karis, T. E. Surface diffusion of thin perfluoropolyalkylether films. Journal of Applied Physics 1996, 79,5788 .

(46) Min, B. G.; Choi, J. W.; Brown, H. R.; Yoon, D. Y.; O'Connor, T. M.; Jhon, M. S. Spreading characteristics of thin liquid films of perfluoropolyalkylethers on solid surfaces. Effects of chain-end functionality and humidity. Tribology Letters 1995, 1, 225232.

(47) Valignat, M. P.; Oshanin, G.; Villette, S.; Cazabat, A. M.; Moreau, M. Molecular weight dependence of spreading rates of ultrathin polymeric films. Physical Review Letters 1998, 80, 5377-5380.

(48) Joanny, J. F. Dynamics of wetting: Interface profile of a spreading liquid. Journal de mécanique théorique et appliquée 1986, 249-271. 
(49) Joanny, J. F.; De Gennes, P. G. Upward creep of a wetting fluid: a scaling analysis. Journal de Physique 1986, 4\%.1, 121-127.

(50) Novotny, V. J. Migration of liquid polymers on solid surfaces. The Journal of Chemical Physics 1990, 92, 3189-3196.

(51) Albrecht, U.; Otto, A.; Leiderer, P. 2-Dimensional Liquid Polymer Diffusion - Experiment and Simulation. Physical Review Letters 1992, 68, 3192-3195.

(52) Albrecht, U.; Otto, A.; Leiderer, P. Diffusive 2-Dimensional Spreading of a Polymer. Surface Science 1993, 283, 383-386.

(53) AlaNissila, T.; Herminghaus, S.; Hjelt, T.; Leiderer, P. Diffusive spreading of chainlike molecules on surfaces. Physical Review Letters 1996, 76, 4003-4006. 